\title{
About Graph Complements
}

\author{
Sebastian Koch \\ Johannes Gutenberg University \\ Mainz, Germany ${ }^{1}$
}

\begin{abstract}
Summary. This article formalizes different variants of the complement graph in the Mizar system [3, based on the formalization of graphs in [6].

MSC: $05 \mathrm{C} 76$ 68V20
\end{abstract}

Keywords: graph complement; loop

MML identifier: GLIB_012, version: 8.1.09 5.60.1371

\section{INTRODUCTION}

In the first section of this article, the property of a graph to be reflexive is rigorously introduced. But since the irreflexive attribute was called loopless in 6], loopfull was chosen this time.

The following section introduces a mode to add loops to a subset of the vertices of a graph. It is shown that for a finite subset this operation can be done by adding a loop at a time (cf. [5]). It is also shown that adding loops can preserve isomorphism between graphs, if the subset of vertices of the second graph the loops are added to is the image under an isomorphism of the subset of vertices of the first graphs the loops are added to.

The next four sections formalize the directed complement with loops, the undirected complement with loops, the directed complement without loops and the undirected complement without loops, respectively. Given a simple undirected graph, its complement is usually defined on the same vertex set; two different vertices being adjacent iff they weren't adjacent in the original graph

\footnotetext{
${ }^{1}$ The author is enrolled in the Johannes Gutenberg University in Mayence, Germany, mailto: skoch02@students.uni-mainz.de 
[8], 2], [1]. A similar definition can be given for simple digraphs [1. The loop variants are introduced on the base of similarity between graphs and relations.

In contrast to the literature the definitions formalized allow to take the complement of any graphs, with parallel edges simply being ignored. So any complement of a graph is also a complement of that graph with its parallel edges removed. Furthermore on a technical note, the vertex sets of the graph and its complement are required to be the same, while the edge sets have to be disjoint. This choice was made to ensure the union of a graph and its complement would be complete and its intersection edgeless. Since the edge set of the complement graph is otherwise unspecified, for each complement type all possible complements of a graph are only isomorphic to each other. Other theorems include:

- Involutiveness of the graph complement: If a graph is of the right type (e.g. simple for undirected complement without loops), then it is the complement of its complement.

- The complement of an edgeless graph is complete.

- The undirected complement without loops of a complete graph is edgeless.

- The complement of an unconnected graph is connected.

- The neighbors of a vertex in a complement without loops of a graph is the complement of the neighbors in the original graph.

- If a graph has order at least 3 , no vertex can be an endvertex in both that graph and its directed complement without loops (the directed $K_{2}$ is a counterexample for order equal to 2.)

- If a graph has order at least 4 , no vertex can be an endvertex in both that graph and its undirected complement without loops $\left(P_{3}\right.$ with its complement $K_{2}+K_{1}$ is a counterexample for order equal to 3 .)

The last section briefly introduces the property of a graph to be self-complementary for all four variants, but without going into depth. However, it is shown that these four variants are mutually exclusive, except for $K_{1}$ which is selfcomplementary with respect to the directed or undirected complement, without loops in both cases. 


\section{LoOpfull GraphS}

Let $G$ be a graph. We say that $G$ is loopfull if and only if

(Def. 1) for every vertex $v$ of $G$, there exists an object $e$ such that $e$ joins $v$ and $v$ in $G$.

Let us consider a graph $G$. Now we state the propositions:

(1) $G$ is loopfull if and only if for every vertex $v$ of $G$, there exists an object $e$ such that $e$ joins $v$ to $v$ in $G$.

(2) $G$ is loopfull if and only if for every vertex $v$ of $G, v$ and $v$ are adjacent.

One can verify that every graph which is loopfull is also non loopless and every graph which is trivial and non loopless is also loopfull and there exists a graph which is loopfull and complete and there exists a graph which is non loopfull.

Now we state the proposition:

(3) Let us consider a graph $G_{1}$, a set $E$, and a graph $G_{2}$ given by reversing directions of the edges $E$ of $G_{1}$. Then $G_{1}$ is loopfull if and only if $G_{2}$ is loopfull.

Let $G$ be a loopfull graph and $E$ be a set. One can check that every graph given by reversing directions of the edges $E$ of $G$ is loopfull.

Let $G$ be a non loopfull graph. Let us observe that every graph given by reversing directions of the edges $E$ of $G$ is non loopfull.

Now we state the propositions:

(4) Let us consider graphs $G_{1}, G_{2}$. If $G_{1} \approx G_{2}$, then if $G_{1}$ is loopfull, then $G_{2}$ is loopfull.

(5) Let us consider a loopfull graph $G_{2}$, and a supergraph $G_{1}$ of $G_{2}$. Suppose the vertices of $G_{1}=$ the vertices of $G_{2}$. Then $G_{1}$ is loopfull.

Let us consider graphs $G_{1}, G_{2}$ and a partial graph mapping $F$ from $G_{1}$ to $G_{2}$. Now we state the propositions:

(6) Suppose $\operatorname{rng} F_{\mathbb{V}}=$ the vertices of $G_{2}$ and $G_{1} \cdot \operatorname{loops}() \subseteq \operatorname{dom}\left(F_{\mathbb{E}}\right)$. Then if $G_{1}$ is loopfull, then $G_{2}$ is loopfull.

(7) If $F$ is total and onto, then if $G_{1}$ is loopfull, then $G_{2}$ is loopfull. The theorem is a consequence of (6).

(8) Suppose $F$ is semi-continuous and $\operatorname{dom}\left(F_{\mathbb{V}}\right)=$ the vertices of $G_{1}$ and $G_{2} \cdot \operatorname{loops}() \subseteq \operatorname{rng} F_{\mathbb{E}}$. Then if $G_{2}$ is loopfull, then $G_{1}$ is loopfull.

(9) If $F$ is total, onto, and semi-continuous, then if $G_{2}$ is loopfull, then $G_{1}$ is loopfull. The theorem is a consequence of (8).

(10) If $F$ is isomorphism, then $G_{1}$ is loopfull iff $G_{2}$ is loopfull. 
Let $G$ be a loopfull graph and $V$ be a set. Let us observe that every subgraph of $G$ induced by $V$ is loopfull and every subgraph of $G$ with vertices $V$ removed is loopfull and every subgraph of $G$ with vertex $V$ removed is loopfull.

Let $G$ be a non loopfull graph. Let us observe that every spanning subgraph of $G$ is non loopfull.

Let $E$ be a set. Let us note that every subgraph of $G$ induced by the vertices of $G$ and $E$ is non loopfull and every subgraph of $G$ with edges $E$ removed is non loopfull and every subgraph of $G$ with edge $E$ removed is non loopfull.

Now we state the proposition:

(11) Let us consider a graph $G_{2}$, a set $V$, and a supergraph $G_{1}$ of $G_{2}$ extended by the vertices from $V$. Suppose $V \backslash$ (the vertices of $\left.G_{2}\right) \neq \emptyset$. Then $G_{1}$ is not loopfull.

Let $G$ be a non loopfull graph and $V$ be a set. Observe that every supergraph of $G$ extended by the vertices from $V$ is non loopfull.

Let $G$ be a loopfull graph and $v, e, w$ be objects. One can verify that every supergraph of $G$ extended by $e$ between vertices $v$ and $w$ is loopfull.

Now we state the propositions:

(12) Let us consider a graph $G_{2}$, a vertex $v$ of $G_{2}$, objects $e, w$, and a supergraph $G_{1}$ of $G_{2}$ extended by $v, w$ and $e$ between them. Suppose $e \notin$ the edges of $G_{2}$ and $w \notin$ the vertices of $G_{2}$. Then $G_{1}$ is not loopfull.

(13) Let us consider a graph $G_{2}$, objects $v, e$, a vertex $w$ of $G_{2}$, and a supergraph $G_{1}$ of $G_{2}$ extended by $v, w$ and $e$ between them. Suppose $e \notin$ the edges of $G_{2}$ and $v \notin$ the vertices of $G_{2}$. Then $G_{1}$ is not loopfull.

Let $G$ be a non loopfull graph and $v, e, w$ be objects. Let us observe that every supergraph of $G$ extended by $v, w$ and $e$ between them is non loopfull.

Now we state the proposition:

(14) Let us consider a graph $G_{2}$, an object $v$, a subset $V$ of the vertices of $G_{2}$, and a supergraph $G_{1}$ of $G_{2}$ extended by vertex $v$ and edges between $v$ and $V$ of $G_{2}$. Suppose $v \notin$ the vertices of $G_{2}$. Then $G_{1}$ is not loopfull.

Let $G$ be a non loopfull graph, $v$ be an object, and $V$ be a set. One can check that every supergraph of $G$ extended by vertex $v$ and edges between $v$ and $V$ of $G$ is non loopfull.

Let $G$ be a loopfull graph. Let us note that every subgraph of $G$ with parallel edges removed is loopfull and every subgraph of $G$ with directed-parallel edges removed is loopfull.

Let $G$ be a non loopfull graph. Note that every subgraph of $G$ with parallel edges removed is non loopfull and every subgraph of $G$ with directed-parallel edges removed is non loopfull. 
Let $G_{F}$ be a graph-yielding function. We say that $G_{F}$ is loopfull if and only if

(Def. 2) for every object $x$ such that $x \in \operatorname{dom} G_{F}$ there exists a graph $G$ such that $G_{F}(x)=G$ and $G$ is loopfull.

Let $G$ be a loopfull graph. Let us note that $\langle G\rangle$ is loopfull and $\mathbb{N} \longmapsto G$ is loopfull.

Let $G_{F}$ be a non empty, graph-yielding function. Note that $G_{F}$ is loopfull if and only if the condition (Def. 3) is satisfied.

(Def. 3) for every element $x$ of $\operatorname{dom} G_{F}, G_{F}(x)$ is loopfull.

Let $G_{S q}$ be a graph sequence. Let us note that $G_{S q}$ is loopfull if and only if the condition (Def. 4) is satisfied.

(Def. 4) for every natural number $n, G_{S q}(n)$ is loopfull.

Let us observe that every graph-yielding function which is empty is also loopfull and every graph-yielding function which is non empty and loopfull is also non loopless and there exists a graph sequence which is loopfull and there exists a graph-yielding finite sequence which is non empty and loopfull.

Let $G_{F}$ be a loopfull, non empty, graph-yielding function and $x$ be an element of $\operatorname{dom} G_{F}$. Note that $G_{F}(x)$ is loopfull.

Let $G_{S q}$ be a loopfull graph sequence and $x$ be a natural number. Note that $G_{S q}(x)$ is loopfull.

Let $p$ be a loopfull, graph-yielding finite sequence and $n$ be a natural number. Observe that $p\left\lceil n\right.$ is loopfull and $p_{\lfloor n}$ is loopfull.

Let $m$ be a natural number. One can check that $\operatorname{smid}(p, m, n)$ is loopfull and $\langle p(m), \ldots, p(n)\rangle$ is loopfull.

Let $p, q$ be loopfull, graph-yielding finite sequences. Observe that $p^{\frown} q$ is loopfull and $p \wedge q$ is loopfull.

Let $G_{1}, G_{2}$ be loopfull graphs. Note that $\left\langle G_{1}, G_{2}\right\rangle$ is loopfull.

Let $G_{3}$ be a loopfull graph. Let us note that $\left\langle G_{1}, G_{2}, G_{3}\right\rangle$ is loopfull.

\section{Adding Loops to a Graph}

Let $G$ be a graph and $V$ be a set.

A graph by adding a loop to each vertex of $G$ in $V$ is a supergraph of $G$ defined by

(Def. 5) (i) the vertices of $i t=$ the vertices of $G$ and there exists a set $E$ and there exists a one-to-one function $f$ such that $E$ misses the edges of $G$ and the edges of $i t=$ (the edges of $G) \cup E$ and $\operatorname{dom} f=E$ and $\operatorname{rng} f=V$ and the source of $i t=($ the source of $G)+\cdot f$ and the target of $i t=($ the target of $G)+\cdot f$, if $V \subseteq$ the vertices of $G$, 
(ii) it $\approx G$, otherwise.

A graph by adding a loop to each vertex of $G$ is a graph by adding a loop to each vertex of $G$ in the vertices of $G$. Now we state the proposition:

(15) Let us consider a graph $G_{2}$, a set $V$, and a graph $G_{1}$ by adding a loop to each vertex of $G_{2}$ in $V$. Then the vertices of $G_{1}=$ the vertices of $G_{2}$.

Let us consider a graph $G_{2}$, a set $V$, a graph $G_{1}$ by adding a loop to each vertex of $G_{2}$ in $V$, and objects $e, v, w$. Now we state the propositions:

(16) If $v \neq w$, then $e$ joins $v$ to $w$ in $G_{1}$ iff $e$ joins $v$ to $w$ in $G_{2}$.

(17) If $v \neq w$, then $e$ joins $v$ and $w$ in $G_{1}$ iff $e$ joins $v$ and $w$ in $G_{2}$. The theorem is a consequence of (16).

(18) Let us consider a graph $G_{2}$, a subset $V$ of the vertices of $G_{2}$, a graph $G_{1}$ by adding a loop to each vertex of $G_{2}$ in $V$, and a vertex $v$ of $G_{1}$. If $v \in V$, then $v$ and $v$ are adjacent.

(19) Let us consider a graph $G_{2}$, a set $V$, and a graph $G_{1}$ by adding a loop to each vertex of $G_{2}$ in $V$. Then $G_{1}$.order ()$=G_{2}$.order () .

(20) Let us consider a graph $G_{2}$, a subset $V$ of the vertices of $G_{2}$, and a graph $G_{1}$ by adding a loop to each vertex of $G_{2}$ in $V$. Then $G_{1} \cdot \operatorname{size}()=G_{2} \cdot \operatorname{size}()+$ $\overline{\bar{V}}$.

(21) Let us consider graphs $G_{1}, G_{2}$. Then $G_{1}$ is a graph by adding a loop to each vertex of $G_{2}$ in $\emptyset$ if and only if $G_{1} \approx G_{2}$. The theorem is a consequence of $(15)$.

(22) Every graph is a graph by adding a loop to each vertex of $G$ in $\emptyset$.

(23) Let us consider a graph $G$, subsets $V_{1}, V_{2}$ of the vertices of $G$, a graph $G_{1}$ by adding a loop to each vertex of $G$ in $V_{1}$, and a graph $G_{2}$ by adding a loop to each vertex of $G_{1}$ in $V_{2}$. Suppose $V_{1}$ misses $V_{2}$. Then $G_{2}$ is a graph by adding a loop to each vertex of $G$ in $V_{1} \cup V_{2}$. The theorem is a consequence of (15).

(24) Let us consider a graph $G_{3}$, subsets $V_{1}, V_{2}$ of the vertices of $G_{3}$, and a graph $G_{1}$ by adding a loop to each vertex of $G_{3}$ in $V_{1} \cup V_{2}$. Suppose $V_{1}$ misses $V_{2}$. Then there exists a graph $G_{2}$ by adding a loop to each vertex of $G_{3}$ in $V_{1}$ such that $G_{1}$ is a graph by adding a loop to each vertex of $G_{2}$ in $V_{2}$.

(25) Let us consider a loopless graph $G_{2}$, a subset $V$ of the vertices of $G_{2}$, and a graph $G_{1}$ by adding a loop to each vertex of $G_{2}$ in $V$. Then

(i) the edges of $G_{2}$ misses $G_{1} \cdot \operatorname{loops}()$, and

(ii) the edges of $G_{1}=\left(\right.$ the edges of $\left.G_{2}\right) \cup G_{1} \cdot \operatorname{loops}()$. 
(26) Let us consider a loopless graph $G_{1}$, a set $V$, a graph $G_{2}$ by adding a loop to each vertex of $G_{1}$ in $V$, and a subgraph $G_{3}$ of $G_{2}$ with loops removed. Then $G_{1} \approx G_{3}$. The theorem is a consequence of (25).

(27) Let us consider graphs $G_{1}, G_{2}$, and a vertex $v$ of $G_{2}$. Then $G_{1}$ is a graph by adding a loop to each vertex of $G_{2}$ in $\{v\}$ if and only if there exists an object $e$ such that $e \notin$ the edges of $G_{2}$ and $G_{1}$ is a supergraph of $G_{2}$ extended by $e$ between vertices $v$ and $v$.

(28) Let us consider a graph $G_{2}$, a finite subset $V$ of the vertices of $G_{2}$, and a graph $G_{1}$ by adding a loop to each vertex of $G_{2}$ in $V$. Then there exists a non empty, graph-yielding finite sequence $p$ such that

(i) $p(1) \approx G_{2}$, and

(ii) $p($ len $p)=G_{1}$, and

(iii) len $p=\overline{\bar{V}}+1$, and

(iv) for every element $n$ of $\operatorname{dom} p$ such that $n \leqslant \operatorname{len} p-1$ there exists a vertex $v$ of $G_{2}$ and there exists an object $e$ such that $p(n+1)$ is a supergraph of $p(n)$ extended by $e$ between vertices $v$ and $v$ and $v \in V$ and $e \notin$ the edges of $p(n)$.

Proof: Define $\mathcal{P}$ [natural number] $\equiv$ for every graph $G_{2}$ for every finite subset $V$ of the vertices of $G_{2}$ for every graph $G_{1}$ by adding a loop to each vertex of $G_{2}$ in $V$ such that $\overline{\bar{V}}=\$_{1}$ there exists a non empty, graphyielding finite sequence $p$ such that $p(1) \approx G_{2}$ and $p(\operatorname{len} p)=G_{1}$ and len $p=\overline{\bar{V}}+1$.

For every element $n$ of $\operatorname{dom} p$ such that $n \leqslant \operatorname{len} p-1$ there exists a vertex $v$ of $G_{2}$ and there exists an object $e$ such that $p(n+1)$ is a supergraph of $p(n)$ extended by $e$ between vertices $v$ and $v$ and $v \in V$ and $e \notin$ the edges of $p(n) . \mathcal{P}[0]$. For every natural number $k$ such that $\mathcal{P}[k]$ holds $\mathcal{P}[k+1]$. For every natural number $k, \mathcal{P}[k]$.

(29) Let us consider graphs $G_{3}, G_{4}$, sets $V_{1}, V_{2}$, a graph $G_{1}$ by adding a loop to each vertex of $G_{3}$ in $V_{1}$, a graph $G_{2}$ by adding a loop to each vertex of $G_{4}$ in $V_{2}$, and a partial graph mapping $F_{0}$ from $G_{3}$ to $G_{4}$. Suppose $V_{1} \subseteq$ the vertices of $G_{3}$ and $V_{2} \subseteq$ the vertices of $G_{4}$ and $F_{0 \mathbb{V}} \backslash V_{1}$ is oneto-one and $\operatorname{dom}\left(F_{0 \mathbb{V}}\left\lceil V_{1}\right)=V_{1}\right.$ and $\operatorname{rng}\left(F_{0 \mathbb{V}}\left\lceil V_{1}\right)=V_{2}\right.$. Then there exists a partial graph mapping $F$ from $G_{1}$ to $G_{2}$ such that

(i) $F_{\mathbb{V}}=F_{0 \mathbb{V}}$, and

(ii) $F_{\mathbb{E}}\left\lceil\operatorname{dom}\left(F_{0 \mathbb{E}}\right)=F_{0 \mathbb{E}}\right.$, and

(iii) if $F_{0}$ is not empty, then $F$ is not empty, and

(iv) if $F_{0}$ is total, then $F$ is total, and 
(v) if $F_{0}$ is onto, then $F$ is onto, and

(vi) if $F_{0}$ is one-to-one, then $F$ is one-to-one, and

(vii) if $F_{0}$ is directed, then $F$ is directed, and

(viii) if $F_{0}$ is weak subgraph embedding, then $F$ is weak subgraph embedding, and

(ix) if $F_{0}$ is isomorphism, then $F$ is isomorphism, and

(x) if $F_{0}$ is directed-isomorphism, then $F$ is directed-isomorphism.

Proof: Reconsider $f=F_{0 \mathbb{V}}$ as a partial function from the vertices of $G_{1}$ to the vertices of $G_{2}$. Consider $E_{1}$ being a set, $f_{1}$ being a one-to-one function such that $E_{1}$ misses the edges of $G_{3}$ and the edges of $G_{1}=$ (the edges of $\left.G_{3}\right) \cup E_{1}$ and $\operatorname{dom} f_{1}=E_{1}$ and $\operatorname{rng} f_{1}=V_{1}$ and the source of $G_{1}=$ (the source of $\left.G_{3}\right)+\cdot f_{1}$ and the target of $G_{1}=\left(\right.$ the target of $\left.G_{3}\right)+\cdot f_{1}$.

Consider $E_{2}$ being a set, $f_{2}$ being a one-to-one function such that $E_{2}$ misses the edges of $G_{4}$ and the edges of $G_{2}=$ (the edges of $\left.G_{4}\right) \cup E_{2}$ and $\operatorname{dom} f_{2}=E_{2}$ and $\operatorname{rng} f_{2}=V_{2}$ and the source of $G_{2}=$ (the source of $\left.G_{4}\right)+\cdot f_{2}$ and the target of $G_{2}=$ (the target of $\left.G_{4}\right)+\cdot f_{2}$. Set $h=f_{2}{ }^{-1}$. $\left(F_{0 \mathbb{V}} \backslash V_{1}\right) \cdot f_{1}$. Set $g=F_{0 \mathbb{E}}+\cdot h$. Reconsider $F=\langle f, g\rangle$ as a partial graph mapping from $G_{1}$ to $G_{2}$. If $F_{0}$ is total, then $F$ is total. If $F_{0}$ is onto, then $F$ is onto by [7, (6)]. If $F_{0}$ is one-to-one, then $F$ is one-to-one. If $F_{0}$ is directed, then $F$ is directed by [4, (70), (71)].

(30) Let us consider a graph $G_{3}$, a $G_{3}$-isomorphic graph $G_{4}$, and a graph $G_{1}$ by adding a loop to each vertex of $G_{3}$. Then every graph by adding a loop to each vertex of $G_{4}$ is $G_{1}$-isomorphic. The theorem is a consequence of (29).

(31) Let us consider a graph $G_{3}$, a $G_{3}$-directed-isomorphic graph $G_{4}$, and a graph $G_{1}$ by adding a loop to each vertex of $G_{3}$. Then every graph by adding a loop to each vertex of $G_{4}$ is $G_{1}$-directed-isomorphic. The theorem is a consequence of (29).

(32) Let us consider graphs $G_{3}, G_{4}$, a set $V$, a graph $G_{1}$ by adding a loop to each vertex of $G_{3}$ in $V$, and a graph $G_{2}$ by adding a loop to each vertex of $G_{4}$ in $V$. If $G_{3} \approx G_{4}$, then $G_{2}$ is $G_{1}$-directed-isomorphic. The theorem is a consequence of (29).

(33) Let us consider a graph $G_{3}$, sets $V, E$, a graph $G_{4}$ given by reversing directions of the edges $E$ of $G_{3}$, and a graph $G_{1}$ by adding a loop to each vertex of $G_{3}$ in $V$. Then every graph by adding a loop to each vertex of $G_{4}$ in $V$ is $G_{1}$-isomorphic. The theorem is a consequence of (29).

(34) Let us consider a graph $G_{3}$, sets $E, V$, a graph $G_{4}$ given by reversing directions of the edges $E$ of $G_{3}$, a graph $G_{1}$ by adding a loop to each vertex 
of $G_{3}$ in $V$, and a graph $G_{2}$ given by reversing directions of the edges $E$ of $G_{1}$. Suppose $E \subseteq$ the edges of $G_{3}$. Then $G_{2}$ is a graph by adding a loop to each vertex of $G_{4}$ in $V$. The theorem is a consequence of (15).

(35) Let us consider a graph $G_{3}$, a subset $V_{1}$ of the vertices of $G_{3}$, a non empty subset $V_{2}$ of the vertices of $G_{3}$, a subgraph $G_{4}$ of $G_{3}$ induced by $V_{2}$, and a graph $G_{1}$ by adding a loop to each vertex of $G_{3}$ in $V_{1}$. Then every subgraph of $G_{1}$ induced by $V_{2}$ is a graph by adding a loop to each vertex of $G_{4}$ in $V_{1} \cap V_{2}$.

(36) Let us consider a graph $G_{2}$, a set $V$, a graph $G_{1}$ by adding a loop to each vertex of $G_{2}$ in $V$, a vertex $v_{1}$ of $G_{1}$, and a vertex $v_{2}$ of $G_{2}$. Suppose $v_{1} \notin V$ and $v_{1}=v_{2}$. Then

(i) $v_{1}$ is isolated iff $v_{2}$ is isolated, and

(ii) $v_{1}$ is endvertex iff $v_{2}$ is endvertex.

The theorem is a consequence of (17).

(37) Let us consider a graph $G_{2}$, a set $V$, a graph $G_{1}$ by adding a loop to each vertex of $G_{2}$ in $V$, and a path $P$ of $G_{1}$. Then

(i) $P$ is a path of $G_{2}$, or

(ii) there exist objects $v, e$ such that $e$ joins $v$ and $v$ in $G_{1}$ and $P=$ $G_{1} \cdot \operatorname{walkOf}(v, e, v)$.

The theorem is a consequence of (15).

(38) Let us consider a graph $G_{2}$, a set $V$, a graph $G_{1}$ by adding a loop to each vertex of $G_{2}$ in $V$, and a walk $W$ of $G_{1}$. Suppose $W$.edges() misses $\left(G_{1} \cdot \operatorname{loops}()\right) \backslash\left(G_{2} \cdot \operatorname{loops}()\right)$. Then $W$ is a walk of $G_{2}$. The theorem is a consequence of (15).

Let $G$ be a graph. Observe that every graph by adding a loop to each vertex of $G$ is loopfull.

Let $V$ be a non empty subset of the vertices of $G$. Observe that every graph by adding a loop to each vertex of $G$ in $V$ is non loopless.

Now we state the proposition:

(39) Let us consider a graph $G_{2}$, a set $V$, and a graph $G_{1}$ by adding a loop to each vertex of $G_{2}$ in $V$. Then $G_{1}$ is finite if and only if $G_{2}$ is finite. The theorem is a consequence of (15).

Let $G$ be a finite graph and $V$ be a set. Observe that every graph by adding a loop to each vertex of $G$ in $V$ is finite.

Let $G$ be a non finite graph. Note that every graph by adding a loop to each vertex of $G$ in $V$ is non finite.

Now we state the proposition: 
(40) Let us consider a graph $G_{2}$, a set $V$, and a graph $G_{1}$ by adding a loop to each vertex of $G_{2}$ in $V$. Then $G_{1}$ is connected if and only if $G_{2}$ is connected. The theorem is a consequence of (15) and (37).

Let $G$ be a connected graph and $V$ be a set. Let us observe that every graph by adding a loop to each vertex of $G$ in $V$ is connected.

Let $G$ be a non connected graph. Let us note that every graph by adding a loop to each vertex of $G$ in $V$ is non connected.

Now we state the proposition:

(41) Let us consider a graph $G_{2}$, a set $V$, and a graph $G_{1}$ by adding a loop to each vertex of $G_{2}$ in $V$. Then $G_{1}$ is chordal if and only if $G_{2}$ is chordal. The theorem is a consequence of (17) and (37).

Let $G$ be a chordal graph and $V$ be a set. Let us observe that every graph by adding a loop to each vertex of $G$ in $V$ is chordal.

Let $G$ be a non edgeless graph. Let us note that every graph by adding a loop to each vertex of $G$ in $V$ is non edgeless.

Let $G$ be a loopfull graph. Note that every graph by adding a loop to each vertex of $G$ in $V$ is loopfull.

Let $G$ be a simple graph. Let us note that every graph by adding a loop to each vertex of $G$ in $V$ is non-multi.

Let $G$ be a directed-simple graph. Note that every graph by adding a loop to each vertex of $G$ in $V$ is non-directed-multi.

Let us consider a graph $G_{2}$, a subset $V$ of the vertices of $G_{2}$, a graph $G_{1}$ by adding a loop to each vertex of $G_{2}$ in $V$, a vertex $v_{1}$ of $G_{1}$, and a vertex $v_{2}$ of $G_{2}$. Now we state the propositions:

(42) Suppose $v_{1}=v_{2}$ and $v_{1} \in V$. Then there exists an object $e$ such that

(i) $e$ joins $v_{1}$ to $v_{1}$ in $G_{1}$, and

(ii) $e \notin$ the edges of $G_{2}$, and

(iii) $v_{1} \cdot \operatorname{edges} \operatorname{In}()=v_{2} \cdot \operatorname{edges} \operatorname{In}() \cup\{e\}$, and

(iv) $v_{1}$.edgesOut ()$=v_{2}$.edgesOut ()$\cup\{e\}$, and

(v) $v_{1}$.edgesInOut ()$=v_{2}$.edgesInOut ()$\cup\{e\}$.

(43) If $v_{1}=v_{2}$ and $v_{1} \in V$, then $v_{1}$.inDegree ()$=v_{2}$.inDegree ()$+1$ and $v_{1}$.outDegree ()$=v_{2}$.outDegree ()$+1$ and $v_{1}$.degree ()$=v_{2}$.degree ()$+2$. The theorem is a consequence of (42).

(44) Suppose $v_{1}=v_{2}$ and $v_{1} \notin V$. Then

(i) $v_{1} \cdot$ edges $\operatorname{In}()=v_{2} \cdot \operatorname{edges} \operatorname{In}()$, and

(ii) $v_{1}$.inDegree ()$=v_{2}$.inDegree () , and

(iii) $v_{1}$.edgesOut ()$=v_{2}$.edgesOut () , and 
(iv) $v_{1}$.outDegree ()$=v_{2}$.outDegree () , and

(v) $v_{1}$.edgesInOut ()$=v_{2}$.edgesInOut () , and

(vi) $v_{1}$.degree ()$=v_{2}$.degree () .

\section{Directed Graph Complement with Loops}

Let $G$ be a graph.

A directed graph complement of $G$ with loops is a non-directed-multi graph defined by

(Def. 6) the vertices of $i t=$ the vertices of $G$ and the edges of $i t$ misses the edges of $G$ and for every vertices $v, w$ of $G$, there exists an object $e_{1}$ such that $e_{1}$ joins $v$ to $w$ in $G$ iff there exists no object $e_{2}$ such that $e_{2}$ joins $v$ to $w$ in $i t$.

Now we state the proposition:

(45) Let us consider graphs $G_{1}, G_{2}, G_{3}$, and a directed graph complement $G_{4}$ of $G_{1}$ with loops. Suppose $G_{1} \approx G_{2}$ and $G_{3} \approx G_{4}$. Then $G_{3}$ is a directed graph complement of $G_{2}$ with loops.

Let $G$ be a graph. Observe that there exists a directed graph complement of $G$ with loops which is plain.

Now we state the propositions:

(46) Let us consider a graph $G_{1}$, a directed graph complement $G_{2}$ of $G_{1}$ with loops, and objects $e_{1}, e_{2}, v, w$. If $e_{1}$ joins $v$ to $w$ in $G_{1}$, then $e_{2}$ does not join $v$ to $w$ in $G_{2}$.

(47) Let us consider a graph $G_{1}$, and a subgraph $G_{2}$ of $G_{1}$ with directedparallel edges removed. Then every directed graph complement of $G_{1}$ with loops is a directed graph complement of $G_{2}$ with loops. The theorem is a consequence of (46).

(48) Let us consider graphs $G_{1}, G_{2}$, a subgraph $G_{3}$ of $G_{1}$ with directedparallel edges removed, a subgraph $G_{4}$ of $G_{2}$ with directed-parallel edges removed, a directed graph complement $G_{5}$ of $G_{1}$ with loops, and a directed graph complement $G_{6}$ of $G_{2}$ with loops. Suppose $G_{4}$ is $G_{3}$-directedisomorphic. Then $G_{6}$ is $G_{5}$-directed-isomorphic. The theorem is a consequence of (47).

(49) Let us consider a graph $G_{1}$, a $G_{1}$-directed-isomorphic graph $G_{2}$, and a directed graph complement $G_{3}$ of $G_{1}$ with loops. Then every directed graph complement of $G_{2}$ with loops is $G_{3}$-directed-isomorphic. The theorem is a consequence of (48). 
(50) Let us consider a graph $G_{1}$, and directed graph complements $G_{2}, G_{3}$ of $G_{1}$ with loops. Then $G_{3}$ is $G_{2}$-directed-isomorphic. The theorem is a consequence of (49).

(51) Let us consider a graph $G_{1}$, a graph $G_{2}$ given by reversing directions of the edges of $G_{1}$, and a directed graph complement $G_{3}$ of $G_{1}$ with loops. Then every graph given by reversing directions of the edges of $G_{3}$ is a directed graph complement of $G_{2}$ with loops. The theorem is a consequence of (46).

(52) Let us consider a graph $G_{1}$, a non empty subset $V$ of the vertices of $G_{1}$, a subgraph $G_{2}$ of $G_{1}$ induced by $V$, and a directed graph complement $G_{3}$ of $G_{1}$ with loops. Then every subgraph of $G_{3}$ induced by $V$ is a directed graph complement of $G_{2}$ with loops. The theorem is a consequence of (46).

(53) Let us consider a graph $G_{1}$, a proper subset $V$ of the vertices of $G_{1}$, a subgraph $G_{2}$ of $G_{1}$ with vertices $V$ removed, and a directed graph complement $G_{3}$ of $G_{1}$ with loops. Then every subgraph of $G_{3}$ with vertices $V$ removed is a directed graph complement of $G_{2}$ with loops. The theorem is a consequence of $(52)$.

(54) Let us consider a non-directed-multi graph $G_{1}$, and a directed graph complement $G_{2}$ of $G_{1}$ with loops. Then $G_{1}$ is a directed graph complement of $G_{2}$ with loops.

Let us consider a graph $G_{1}$ and a directed graph complement $G_{2}$ of $G_{1}$ with loops. Now we state the propositions:

$G_{1}$.order ()$=G_{2}$.order () .

(56) (i) $G_{1}$ is trivial iff $G_{2}$ is trivial, and

(ii) $G_{1}$ is loopfull iff $G_{2}$ is loopless, and

(iii) $G_{1}$ is loopless iff $G_{2}$ is loopfull.

The theorem is a consequence of (55), (1), and (46).

Let $G$ be a trivial graph. One can verify that every directed graph complement of $G$ with loops is trivial. Let $G$ be a non trivial graph. One can check that every directed graph complement of $G$ with loops is non trivial. Let $G$ be a loopfull graph. Note that every directed graph complement of $G$ with loops is loopless.

Let $G$ be a non loopfull graph. Let us note that every directed graph complement of $G$ with loops is non loopless. Let $G$ be a loopless graph. Observe that every directed graph complement of $G$ with loops is loopfull. Let $G$ be a non loopless graph. Let us observe that every directed graph complement of $G$ with loops is non loopfull.

Now we state the proposition: 
(57) Let us consider a graph $G_{1}$, and a directed graph complement $G_{2}$ of $G_{1}$ with loops. Suppose the edges of $G_{1}=G_{1}$.loops(). Then $G_{2}$ is complete.

Let $G$ be an edgeless graph. One can verify that every directed graph complement of $G$ with loops is complete. Let $G$ be a non connected graph. One can check that every directed graph complement of $G$ with loops is connected.

Now we state the propositions:

(58) Let us consider a graph $G_{1}$, a directed graph complement $G_{2}$ of $G_{1}$ with loops, a vertex $v_{1}$ of $G_{1}$, and a vertex $v_{2}$ of $G_{2}$. Suppose $v_{1}=v_{2}$. Then

(i) if $v_{1}$ is isolated, then $v_{2}$ is not isolated, and

(ii) if $v_{1}$ is endvertex, then $v_{2}$ is not endvertex.

(59) Let us consider a graph $G_{1}$, a directed graph complement $G_{2}$ of $G_{1}$ with loops, and vertices $v, w$ of $G_{1}$. Suppose there exists no object $e$ such that $e$ joins $v$ and $w$ in $G_{1}$. Then there exists an object $e$ such that $e$ joins $v$ and $w$ in $G_{2}$.

Proof: There exists no object $e$ such that $e$ joins $v$ to $w$ in $G_{1}$. Consider $e$ being an object such that $e$ joins $v$ to $w$ in $G_{2}$.

Let us consider a graph $G_{1}$, a directed graph complement $G_{2}$ of $G_{1}$ with loops, a vertex $v_{1}$ of $G_{1}$, and a vertex $v_{2}$ of $G_{2}$. Now we state the propositions:

(60) Suppose $v_{1}=v_{2}$. Then

(i) $v_{2}$.inNeighbors ()$=\left(\right.$ the vertices of $\left.G_{2}\right) \backslash\left(v_{1}\right.$.inNeighbors ()$)$, and

(ii) $v_{2}$.outNeighbors ()$=\left(\right.$ the vertices of $\left.G_{2}\right) \backslash\left(v_{1}\right.$.outNeighbors ()$)$.

(61) Suppose $v_{1}=v_{2}$ and $v_{1}$ is isolated. Then

(i) $v_{2}$.inNeighbors ()$=$ the vertices of $G_{2}$, and

(ii) $v_{2}$.outNeighbors ()$=$ the vertices of $G_{2}$, and

(iii) $v_{2}$.allNeighbors ()$=$ the vertices of $G_{2}$.

The theorem is a consequence of (60).

\section{Undirected Graph Complement with Loops}

Let $G$ be a graph.

An undirected graph complement of $G$ with loops is a non-multi graph defined by

(Def. 7) the vertices of $i t=$ the vertices of $G$ and the edges of $i t$ misses the edges of $G$ and for every vertices $v, w$ of $G$, there exists an object $e_{1}$ such that $e_{1}$ joins $v$ and $w$ in $G$ iff there exists no object $e_{2}$ such that $e_{2}$ joins $v$ and $w$ in $i t$. 
Now we state the proposition:

(62) Let us consider graphs $G_{1}, G_{2}, G_{3}$, and an undirected graph complement $G_{4}$ of $G_{1}$ with loops. Suppose $G_{1} \approx G_{2}$ and $G_{3} \approx G_{4}$. Then $G_{3}$ is an undirected graph complement of $G_{2}$ with loops.

Let $G$ be a graph. Note that there exists an undirected graph complement of $G$ with loops which is plain.

Now we state the propositions:

(63) Let us consider a graph $G_{1}$, and a non-multi graph $G_{2}$. Then $G_{2}$ is an undirected graph complement of $G_{1}$ with loops if and only if the vertices of $G_{2}=$ the vertices of $G_{1}$ and the edges of $G_{2}$ misses the edges of $G_{1}$ and for every vertices $v_{1}, w_{1}$ of $G_{1}$ and for every vertices $v_{2}, w_{2}$ of $G_{2}$ such that $v_{1}=v_{2}$ and $w_{1}=w_{2}$ holds $v_{1}$ and $w_{1}$ are adjacent iff $v_{2}$ and $w_{2}$ are not adjacent.

(64) Let us consider a graph $G_{1}$, an undirected graph complement $G_{2}$ of $G_{1}$ with loops, and objects $e_{1}, e_{2}, v, w$. If $e_{1}$ joins $v$ and $w$ in $G_{1}$, then $e_{2}$ does not join $v$ and $w$ in $G_{2}$.

(65) Let us consider a graph $G_{1}$, and a subgraph $G_{2}$ of $G_{1}$ with parallel edges removed. Then every undirected graph complement of $G_{1}$ with loops is an undirected graph complement of $G_{2}$ with loops. The theorem is a consequence of (64).

(66) Let us consider graphs $G_{1}, G_{2}$, a subgraph $G_{3}$ of $G_{1}$ with parallel edges removed, a subgraph $G_{4}$ of $G_{2}$ with parallel edges removed, an undirected graph complement $G_{5}$ of $G_{1}$ with loops, and an undirected graph complement $G_{6}$ of $G_{2}$ with loops. If $G_{4}$ is $G_{3}$-isomorphic, then $G_{6}$ is $G_{5^{-}}$ isomorphic. The theorem is a consequence of (65).

(67) Let us consider a graph $G_{1}$, a $G_{1}$-isomorphic graph $G_{2}$, and an undirected graph complement $G_{3}$ of $G_{1}$ with loops. Then every undirected graph complement of $G_{2}$ with loops is $G_{3}$-isomorphic. The theorem is a consequence of (66).

(68) Let us consider a graph $G_{1}$, and undirected graph complements $G_{2}, G_{3}$ of $G_{1}$ with loops. Then $G_{3}$ is $G_{2}$-isomorphic. The theorem is a consequence of $(67)$.

(69) Let us consider a graph $G_{1}$, a non empty subset $V$ of the vertices of $G_{1}$, a subgraph $G_{2}$ of $G_{1}$ induced by $V$, and an undirected graph complement $G_{3}$ of $G_{1}$ with loops. Then every subgraph of $G_{3}$ induced by $V$ is an undirected graph complement of $G_{2}$ with loops. The theorem is a consequence of $(64)$.

(70) Let us consider a graph $G_{1}$, a proper subset $V$ of the vertices of $G_{1}$, a subgraph $G_{2}$ of $G_{1}$ with vertices $V$ removed, and an undirected graph 
complement $G_{3}$ of $G_{1}$ with loops. Then every subgraph of $G_{3}$ with vertices $V$ removed is an undirected graph complement of $G_{2}$ with loops. The theorem is a consequence of (69).

(71) Let us consider a non-multi graph $G_{1}$, and an undirected graph complement $G_{2}$ of $G_{1}$ with loops. Then $G_{1}$ is an undirected graph complement of $G_{2}$ with loops.

Let us consider a graph $G_{1}$ and an undirected graph complement $G_{2}$ of $G_{1}$ with loops. Now we state the propositions:

(72) $G_{1}$.order ()$=G_{2}$.order () .

(73) (i) $G_{1}$ is trivial iff $G_{2}$ is trivial, and

(ii) $G_{1}$ is loopfull iff $G_{2}$ is loopless, and

(iii) $G_{1}$ is loopless iff $G_{2}$ is loopfull.

The theorem is a consequence of (72) and (64).

Let $G$ be a trivial graph. Observe that every undirected graph complement of $G$ with loops is trivial.

Let $G$ be a non trivial graph. Let us observe that every undirected graph complement of $G$ with loops is non trivial.

Let $G$ be a loopfull graph. One can verify that every undirected graph complement of $G$ with loops is loopless.

Let $G$ be a non loopfull graph. One can check that every undirected graph complement of $G$ with loops is non loopless.

Let $G$ be a loopless graph. Note that every undirected graph complement of $G$ with loops is loopfull.

Let $G$ be a non loopless graph. Let us note that every undirected graph complement of $G$ with loops is non loopfull.

Now we state the proposition:

(74) Let us consider a graph $G_{1}$, and an undirected graph complement $G_{2}$ of $G_{1}$ with loops. Suppose the edges of $G_{1}=G_{1}$ lloops(). Then $G_{2}$ is complete.

Let $G$ be an edgeless graph. Observe that every undirected graph complement of $G$ with loops is complete.

Now we state the proposition:

(75) Let us consider a complete graph $G_{1}$, and an undirected graph complement $G_{2}$ of $G_{1}$ with loops. Then the edges of $G_{2}=G_{2} \cdot \operatorname{loops}()$. The theorem is a consequence of (64).

Let $G$ be a complete, loopfull graph. Observe that every undirected graph complement of $G$ with loops is edgeless. 
Let $G$ be a non connected graph. Note that every undirected graph complement of $G$ with loops is connected.

Let us consider a graph $G_{1}$, an undirected graph complement $G_{2}$ of $G_{1}$ with loops, a vertex $v_{1}$ of $G_{1}$, and a vertex $v_{2}$ of $G_{2}$. Now we state the propositions:

(76) If $v_{1}=v_{2}$, then if $v_{1}$ is isolated, then $v_{2}$ is not isolated and if $v_{1}$ is endvertex, then $v_{2}$ is not endvertex.

(77) If $v_{1}=v_{2}$, then $v_{2}$.allNeighbors ()$=$ (the vertices of $\left.G_{2}\right) \backslash\left(v_{1}\right.$.allNeighbors ()$)$.

(78) If $v_{1}=v_{2}$ and $v_{1}$ is isolated, then $v_{2}$.allNeighbors ()$=$ the vertices of $G_{2}$. The theorem is a consequence of (77).

\section{Directed Graph Complement without Loops}

Let $G$ be a graph.

A directed graph complement of $G$ is a directed-simple graph defined by

(Def. 8) there exists a directed graph complement $G^{\prime}$ of $G$ with loops such that $i t$ is a subgraph of $G^{\prime}$ with loops removed.

Now we state the proposition:

(79) Let us consider graphs $G_{1}, G_{2}, G_{3}$, and a directed graph complement $G_{4}$ of $G_{1}$. Suppose $G_{1} \approx G_{2}$ and $G_{3} \approx G_{4}$. Then $G_{3}$ is a directed graph complement of $G_{2}$. The theorem is a consequence of (45).

Let $G$ be a graph. One can check that there exists a directed graph complement of $G$ which is plain. Now we state the propositions:

(80) Let us consider a graph $G_{1}$, and a directed-simple graph $G_{2}$. Then $G_{2}$ is a directed graph complement of $G_{1}$ if and only if the vertices of $G_{2}=$ the vertices of $G_{1}$ and the edges of $G_{2}$ misses the edges of $G_{1}$ and for every vertices $v, w$ of $G_{1}$ such that $v \neq w$ holds there exists an object $e_{1}$ such that $e_{1}$ joins $v$ to $w$ in $G_{1}$ iff there exists no object $e_{2}$ such that $e_{2}$ joins $v$ to $w$ in $G_{2}$. The theorem is a consequence of (46), (26), and (1).

(81) Let us consider a graph $G_{1}$, a directed graph complement $G_{2}$ of $G_{1}$, and objects $e_{1}, e_{2}, v, w$. If $e_{1}$ joins $v$ to $w$ in $G_{1}$, then $e_{2}$ does not join $v$ to $w$ in $G_{2}$. The theorem is a consequence of (80).

(82) Let us consider a graph $G_{1}$, and a directed-simple graph $G_{2}$ of $G_{1}$. Then every directed graph complement of $G_{1}$ is a directed graph complement of $G_{2}$. The theorem is a consequence of (80) and (81).

(83) Let us consider graphs $G_{1}, G_{2}$, a directed-simple graph $G_{3}$ of $G_{1}$, a directed-simple graph $G_{4}$ of $G_{2}$, a directed graph complement $G_{5}$ of $G_{1}$, 
and a directed graph complement $G_{6}$ of $G_{2}$. Suppose $G_{4}$ is $G_{3}$-directedisomorphic. Then $G_{6}$ is $G_{5}$-directed-isomorphic. The theorem is a consequence of (82) and (80).

(84) Let us consider a graph $G_{1}$, a $G_{1}$-directed-isomorphic graph $G_{2}$, and a directed graph complement $G_{3}$ of $G_{1}$. Then every directed graph complement of $G_{2}$ is $G_{3}$-directed-isomorphic. The theorem is a consequence of (83).

(85) Let us consider a graph $G_{1}$, and directed graph complements $G_{2}, G_{3}$ of $G_{1}$. Then $G_{3}$ is $G_{2}$-directed-isomorphic. The theorem is a consequence of (84).

(86) Let us consider a graph $G_{1}$, a graph $G_{2}$ given by reversing directions of the edges of $G_{1}$, and a directed graph complement $G_{3}$ of $G_{1}$. Then every graph given by reversing directions of the edges of $G_{3}$ is a directed graph complement of $G_{2}$. The theorem is a consequence of (80) and (81).

(87) Let us consider a graph $G_{1}$, a non empty subset $V$ of the vertices of $G_{1}$, a subgraph $G_{2}$ of $G_{1}$ induced by $V$, and a directed graph complement $G_{3}$ of $G_{1}$. Then every subgraph of $G_{3}$ induced by $V$ is a directed graph complement of $G_{2}$. The theorem is a consequence of (80) and (81).

(88) Let us consider a graph $G_{1}$, a proper subset $V$ of the vertices of $G_{1}$, a subgraph $G_{2}$ of $G_{1}$ with vertices $V$ removed, and a directed graph complement $G_{3}$ of $G_{1}$. Then every subgraph of $G_{3}$ with vertices $V$ removed is a directed graph complement of $G_{2}$. The theorem is a consequence of (80) and (87).

(89) Let us consider a directed-simple graph $G_{1}$, and a directed graph complement $G_{2}$ of $G_{1}$. Then $G_{1}$ is a directed graph complement of $G_{2}$. The theorem is a consequence of (80).

Let us consider a graph $G_{1}$ and a directed graph complement $G_{2}$ of $G_{1}$. Now we state the propositions:

(90) $G_{1}$.order ()$=G_{2}$.order () .

(91) $G_{1}$ is trivial if and only if $G_{2}$ is trivial. The theorem is a consequence of (90).

Let $G$ be a trivial graph. One can verify that every directed graph complement of $G$ is trivial. Let $G$ be a non trivial graph. One can check that every directed graph complement of $G$ is non trivial. Now we state the proposition:

(92) Let us consider a graph $G_{1}$, and a directed graph complement $G_{2}$ of $G_{1}$. Suppose the edges of $G_{1}=G_{1} \cdot \operatorname{loops}()$. Then $G_{2}$ is complete. The theorem is a consequence of $(80)$.

Let $G$ be an edgeless graph. One can check that every directed graph com- 
plement of $G$ is complete. Let $G$ be a trivial, edgeless graph. Let us observe that every directed graph complement of $G$ is edgeless. Let $G$ be a non connected graph. One can check that every directed graph complement of $G$ is connected. Now we state the proposition:

(93) Let us consider a non trivial graph $G_{1}$, a directed graph complement $G_{2}$ of $G_{1}$, a vertex $v_{1}$ of $G_{1}$, and a vertex $v_{2}$ of $G_{2}$. If $v_{1}=v_{2}$, then if $v_{1}$ is isolated, then $v_{2}$ is not isolated. The theorem is a consequence of (80).

Let us consider a graph $G_{1}$, a directed graph complement $G_{2}$ of $G_{1}$, a vertex $v_{1}$ of $G_{1}$, and a vertex $v_{2}$ of $G_{2}$. Now we state the propositions:

(94) If $v_{1}=v_{2}$ and $3 \subseteq G_{1}$.order(), then if $v_{1}$ is endvertex, then $v_{2}$ is not endvertex.

Proof: Consider $u, w$ being vertices of $G_{1}$ such that $u \neq v_{1}$ and $w \neq v_{1}$ and $u \neq w$ and $u$ and $v_{1}$ are adjacent and $v_{1}$ and $w$ are not adjacent. There exists no object $e$ such that $e$ joins $v_{1}$ to $w$ in $G_{1}$. Consider $e_{1}$ being an object such that $e_{1}$ joins $v_{1}$ to $w$ in $G_{2}$. There exists no object $e$ such that $e$ joins $w$ to $v_{1}$ in $G_{1}$. Consider $e_{2}$ being an object such that $e_{2}$ joins $w$ to $v_{1}$ in $G_{2}$. Consider $e^{\prime}$ being an object such that $v_{2}$.edgesInOut ()$=\left\{e^{\prime}\right\}$ and $e^{\prime}$ does not join $v_{2}$ and $v_{2}$ in $G_{2}$.

(95) Suppose $v_{1}=v_{2}$. Then

(i) $v_{2}$.inNeighbors ()$=\left(\right.$ the vertices of $\left.G_{2}\right) \backslash\left(v_{1}\right.$.inNeighbors ()$\left.\cup\left\{v_{2}\right\}\right)$, and

(ii) $v_{2}$.outNeighbors ()$=\left(\right.$ the vertices of $\left.G_{2}\right) \backslash\left(v_{1}\right.$.outNeighbors ()$\left.\cup\left\{v_{2}\right\}\right)$.

The theorem is a consequence of (60).

(96) Suppose $v_{1}=v_{2}$ and $v_{1}$ is isolated. Then

(i) $v_{2}$.inNeighbors ()$=\left(\right.$ the vertices of $\left.G_{2}\right) \backslash\left\{v_{2}\right\}$, and

(ii) $v_{2}$.outNeighbors ()$=\left(\right.$ the vertices of $\left.G_{2}\right) \backslash\left\{v_{2}\right\}$, and

(iii) $v_{2}$.allNeighbors ()$=\left(\right.$ the vertices of $\left.G_{2}\right) \backslash\left\{v_{2}\right\}$.

The theorem is a consequence of (95).

\section{Undirected Graph Complement without Loops}

Let $G$ be a graph.

A graph complement of $G$ is a simple graph defined by

(Def. 9) there exists an undirected graph complement $G^{\prime}$ of $G$ with loops such that it is a subgraph of $G^{\prime}$ with loops removed.

Now we state the proposition: 
(97) Let us consider graphs $G_{1}, G_{2}, G_{3}$, and a graph complement $G_{4}$ of $G_{1}$. Suppose $G_{1} \approx G_{2}$ and $G_{3} \approx G_{4}$. Then $G_{3}$ is a graph complement of $G_{2}$. The theorem is a consequence of (62).

Let $G$ be a graph. Observe that there exists a graph complement of $G$ which is plain. Let us consider a graph $G_{1}$ and a simple graph $G_{2}$. Now we state the propositions:

(98) $G_{2}$ is a graph complement of $G_{1}$ if and only if the vertices of $G_{2}=$ the vertices of $G_{1}$ and the edges of $G_{2}$ misses the edges of $G_{1}$ and for every vertices $v, w$ of $G_{1}$ such that $v \neq w$ holds there exists an object $e_{1}$ such that $e_{1}$ joins $v$ and $w$ in $G_{1}$ iff there exists no object $e_{2}$ such that $e_{2}$ joins $v$ and $w$ in $G_{2}$. The theorem is a consequence of (64) and (26).

(99) $G_{2}$ is a graph complement of $G_{1}$ if and only if the vertices of $G_{2}=$ the vertices of $G_{1}$ and the edges of $G_{2}$ misses the edges of $G_{1}$ and for every vertices $v_{1}, w_{1}$ of $G_{1}$ and for every vertices $v_{2}, w_{2}$ of $G_{2}$ such that $v_{1}=v_{2}$ and $w_{1}=w_{2}$ and $v_{1} \neq w_{1}$ holds $v_{1}$ and $w_{1}$ are adjacent iff $v_{2}$ and $w_{2}$ are not adjacent. The theorem is a consequence of (98).

(100) Let us consider a graph $G_{1}$, a graph complement $G_{2}$ of $G_{1}$, and objects $e_{1}, e_{2}, v, w$. If $e_{1}$ joins $v$ and $w$ in $G_{1}$, then $e_{2}$ does not join $v$ and $w$ in $G_{2}$. The theorem is a consequence of (98).

(101) Let us consider a graph $G_{1}$, and a simple graph $G_{2}$ of $G_{1}$. Then every graph complement of $G_{1}$ is a graph complement of $G_{2}$. The theorem is a consequence of (98) and (100).

(102) Let us consider graphs $G_{1}, G_{2}$, a simple graph $G_{3}$ of $G_{1}$, a simple graph $G_{4}$ of $G_{2}$, a graph complement $G_{5}$ of $G_{1}$, and a graph complement $G_{6}$ of $G_{2}$. If $G_{4}$ is $G_{3}$-isomorphic, then $G_{6}$ is $G_{5}$-isomorphic. The theorem is a consequence of (101) and (98).

(103) Let us consider a graph $G_{1}$, a $G_{1}$-isomorphic graph $G_{2}$, and a graph complement $G_{3}$ of $G_{1}$. Then every graph complement of $G_{2}$ is $G_{3}$-isomorphic. The theorem is a consequence of (102).

(104) Let us consider a graph $G_{1}$, and graph complements $G_{2}, G_{3}$ of $G_{1}$. Then $G_{3}$ is $G_{2}$-isomorphic. The theorem is a consequence of (103).

(105) Let us consider a graph $G_{1}$, an object $v$, a subset $V$ of the vertices of $G_{1}$, a supergraph $G_{2}$ of $G_{1}$ extended by vertex $v$ and edges between $v$ and $V$ of $G_{1}$, and a graph complement $G_{3}$ of $G_{1}$. Suppose $v \notin$ the vertices of $G_{1}$ and the edges of $G_{2}$ misses the edges of $G_{3}$. Then there exists a supergraph $G_{4}$ of $G_{3}$ extended by vertex $v$ and edges between $v$ and (the vertices of $\left.G_{1}\right) \backslash V$ of $G_{3}$ such that $G_{4}$ is a graph complement of $G_{2}$. The theorem is a consequence of (98).

(106) Let us consider a graph $G_{1}$, an object $v$, a supergraph $G_{2}$ of $G_{1}$ extended 
by $v$, and a graph complement $G_{3}$ of $G_{1}$. Suppose $v \notin$ the vertices of $G_{1}$. Then there exists a supergraph $G_{4}$ of $G_{3}$ extended by vertex $v$ and edges between $v$ and the vertices of $G_{3}$ such that $G_{4}$ is a graph complement of $G_{2}$. The theorem is a consequence of (98) and (105).

(107) Let us consider a graph $G_{1}$, an object $v$, a supergraph $G_{2}$ of $G_{1}$ extended by vertex $v$ and edges between $v$ and the vertices of $G_{1}$, a graph complement $G_{3}$ of $G_{1}$, and a supergraph $G_{4}$ of $G_{3}$ extended by $v$. Suppose $v \notin$ the vertices of $G_{1}$ and the edges of $G_{2}$ misses the edges of $G_{3}$. Then $G_{4}$ is a graph complement of $G_{2}$. The theorem is a consequence of (105) and (97).

(108) Let us consider a graph $G_{1}$, a non empty subset $V$ of the vertices of $G_{1}$, a subgraph $G_{2}$ of $G_{1}$ induced by $V$, and a graph complement $G_{3}$ of $G_{1}$. Then every subgraph of $G_{3}$ induced by $V$ is a graph complement of $G_{2}$. The theorem is a consequence of (98) and (100).

(109) Let us consider a graph $G_{1}$, a proper subset $V$ of the vertices of $G_{1}$, a subgraph $G_{2}$ of $G_{1}$ with vertices $V$ removed, and a graph complement $G_{3}$ of $G_{1}$. Then every subgraph of $G_{3}$ with vertices $V$ removed is a graph complement of $G_{2}$. The theorem is a consequence of (98) and (108).

(110) Let us consider a simple graph $G_{1}$, and a graph complement $G_{2}$ of $G_{1}$. Then $G_{1}$ is a graph complement of $G_{2}$. The theorem is a consequence of (98).

Let us consider a graph $G_{1}$ and a graph complement $G_{2}$ of $G_{1}$. Now we state the propositions:

(111) $G_{1} \cdot$ order ()$=G_{2}$.order () .

(112) $G_{1}$ is trivial if and only if $G_{2}$ is trivial. The theorem is a consequence of (111).

Let $G$ be a trivial graph. Observe that every graph complement of $G$ is trivial. Let $G$ be a non trivial graph. Let us observe that every graph complement of $G$ is non trivial. Now we state the proposition:

(113) Let us consider a graph $G_{1}$, and a graph complement $G_{2}$ of $G_{1}$. Then

(i) $G_{1}$ is complete iff $G_{2}$ is edgeless, and

(ii) the edges of $G_{1}=G_{1}$.loops() iff $G_{2}$ is complete.

The theorem is a consequence of (99) and (98).

Let $G$ be a complete graph. Observe that every graph complement of $G$ is edgeless.

Let $G$ be a non complete graph. Let us observe that every graph complement of $G$ is non edgeless. 
Let $G$ be an edgeless graph. One can verify that every graph complement of $G$ is complete.

Let $G$ be a non connected graph. One can check that every graph complement of $G$ is connected.

Now we state the propositions:

(114) Let us consider a non trivial graph $G_{1}$, a graph complement $G_{2}$ of $G_{1}$, a vertex $v_{1}$ of $G_{1}$, and a vertex $v_{2}$ of $G_{2}$. If $v_{1}=v_{2}$, then if $v_{1}$ is isolated, then $v_{2}$ is not isolated. The theorem is a consequence of (98).

(115) Let us consider a graph $G_{1}$, a graph complement $G_{2}$ of $G_{1}$, a vertex $v_{1}$ of $G_{1}$, and a vertex $v_{2}$ of $G_{2}$. Suppose $v_{1}=v_{2}$ and $G_{1} \cdot \operatorname{order}()=2$. Then

(i) if $v_{1}$ is endvertex, then $v_{2}$ is isolated, and

(ii) if $v_{1}$ is isolated, then $v_{2}$ is endvertex.

The theorem is a consequence of (111), (98), and (100).

(116) Let us consider a simple graph $G_{1}$, a graph complement $G_{2}$ of $G_{1}$, a vertex $v_{1}$ of $G_{1}$, and a vertex $v_{2}$ of $G_{2}$. Suppose $v_{1}=v_{2}$ and $G_{1}$.order ()$=2$. Then

(i) $v_{1}$ is endvertex iff $v_{2}$ is isolated, and

(ii) $v_{1}$ is isolated iff $v_{2}$ is endvertex.

The theorem is a consequence of (110), (111), and (115).

Let us consider a graph $G_{1}$, a graph complement $G_{2}$ of $G_{1}$, a vertex $v_{1}$ of $G_{1}$, and a vertex $v_{2}$ of $G_{2}$. Now we state the propositions:

(117) If $v_{1}=v_{2}$ and $4 \subseteq G_{1}$.order(), then if $v_{1}$ is endvertex, then $v_{2}$ is not endvertex. The theorem is a consequence of (99).

(118) If $v_{1}=v_{2}$, then $v_{2}$.allNeighbors ()$=\left(\right.$ the vertices of $\left.G_{2}\right) \backslash\left(v_{1}\right.$.allNeighbors ()$\left.\cup\left\{v_{2}\right\}\right)$. The theorem is a consequence of (77).

(119) If $v_{1}=v_{2}$ and $v_{1}$ is isolated, then $v_{2}$.allNeighbors ()$=$ (the vertices of $\left.G_{2}\right) \backslash\left\{v_{2}\right\}$. The theorem is a consequence of (118).

\section{Self-Complementary Graphs}

Let $G$ be a graph. We say that $G$ is self-DLcomplementary if and only if (Def. 10) every directed graph complement of $G$ with loops is $G$-directed-isomorphic.

We say that $G$ is self-Lcomplementary if and only if

(Def. 11) every undirected graph complement of $G$ with loops is $G$-isomorphic.

We say that $G$ is self-Dcomplementary if and only if 
(Def. 12) every directed graph complement of $G$ is $G$-directed-isomorphic.

We say that $G$ is self-complementary if and only if

(Def. 13) every graph complement of $G$ is $G$-isomorphic.

Let us consider a graph $G$. Now we state the propositions:

(120) $G$ is self-DLcomplementary if and only if there exists a directed graph complement $H$ of $G$ with loops such that $H$ is $G$-directed-isomorphic. The theorem is a consequence of (50).

(121) $G$ is self-Lcomplementary if and only if there exists an undirected graph complement $H$ of $G$ with loops such that $H$ is $G$-isomorphic. The theorem is a consequence of (68).

(122) $G$ is self-Dcomplementary if and only if there exists a directed graph complement $H$ of $G$ such that $H$ is $G$-directed-isomorphic. The theorem is a consequence of (85).

(123) $G$ is self-complementary if and only if there exists a graph complement $H$ of $G$ such that $H$ is $G$-isomorphic. The theorem is a consequence of (104).

Let us observe that every graph which is self-DLcomplementary is also non loopless, non loopfull, non-directed-multi, and connected and every graph which is self-Lcomplementary is also non loopless, non loopfull, non-multi, and connected and every graph which is self-Dcomplementary is also directed-simple and connected and every graph which is self-complementary is also simple and connected.

Every graph which is trivial and edgeless is also self-Dcomplementary and self-complementary and every graph which is self-Dcomplementary and self-complementary is also trivial and edgeless and every graph which is selfDLcomplementary is also non trivial, non self-Lcomplementary, non self-Dcomplementary, and non self-complementary and every graph which is self-Lcomplementary is also non trivial, non self-DLcomplementary, non self-Dcomplementary, and non self-complementary and there exists a graph which is selfDcomplementary and self-complementary.

\section{REFERENCES}

[1] John Adrian Bondy and U. S. R. Murty. Graph Theory. Graduate Texts in Mathematics, 244. Springer, New York, 2008. ISBN 978-1-84628-969-9.

[2] Reinhard Diestel. Graphentheorie. Springer-Lehrbuch Masterclass, Heidelberg, 4. aufl. 2010. 3., korr. nachdruck edition, 2012. ISBN 978-3-642-14911-5.

[3] Adam Grabowski, Artur Korniłowicz, and Adam Naumowicz. Four decades of Mizar. Journal of Automated Reasoning, 55(3):191-198, 2015. doi 10.1007/s10817-015-9345-1

[4] Sebastian Koch. About supergraphs. Part I. Formalized Mathematics, 26(2):101-124, 2018. doi $10.2478 /$ forma-2018-0009. 
[5] Sebastian Koch. About supergraphs. Part III. Formalized Mathematics, 27(2):153-179, 2019. doi 10.2478/forma-2019-0016.

[6] Gilbert Lee and Piotr Rudnicki. Alternative graph structures Formalized Mathematics, 13(2):235-252, 2005.

[7] Krzysztof Retel. The class of series - parallel graphs. Part I. Formalized Mathematics, 11 (1):99-103, 2003.

[8] Klaus Wagner. Graphentheorie. B.I-Hochschultaschenbücher; 248. Bibliograph. Inst., Mannheim, 1970. ISBN 3-411-00248-4.

Accepted December 30, 2019 